University of Nebraska - Lincoln

DigitalCommons@University of Nebraska - Lincoln

Virology Papers

Virology, Nebraska Center for

$6-2004$

\title{
CD4 T cell responses to influenza infection
}

Deborah M. Brown

University of Nebraska-Lincoln, dbrown7@unl.edu

Eulogia Roman

Trudeau Institute, Inc.

Susan L. Swain

Trudeau Institute, Inc.

Follow this and additional works at: https://digitalcommons.unl.edu/virologypub

Part of the Virology Commons

Brown, Deborah M.; Roman, Eulogia; and Swain, Susan L., "CD4 T cell responses to influenza infection" (2004). Virology Papers. 124.

https://digitalcommons.unl.edu/virologypub/124

This Article is brought to you for free and open access by the Virology, Nebraska Center for at DigitalCommons@University of Nebraska - Lincoln. It has been accepted for inclusion in Virology Papers by an authorized administrator of DigitalCommons@University of Nebraska - Lincoln. 
Published in Seminars in Immunology 16: 3 (June 2004), pp. 171-177 ; doi 10.1016/j.smim.2004.02.004 Copyright (C) 2004 Elsevier

Ltd. Used by permission. http://www.sciencedirect.com/science/journal/10445323

Published online April 8, 2004.

\title{
CD4 T cell responses to influenza infection
}

\author{
Deborah M. Brown, Eulogia Román, and Susan L. Swain \\ Trudeau Institute, Inc., 154 Algonquin Ave., Saranac Lake, NY 12983, USA \\ Corresponding author-S. L. Swain, tel 518 891-3080, fax 518 891-5126, email sswain@northnet.org
}

\begin{abstract}
Immune responses to viral infections involve a complex orchestration between innate signals and adaptive responses of specific $\mathrm{T}$ and B cells. Anti-viral CD4 cells can direct CD8 responses by secreting a Type 1 panel of cytokines including IFN- $\gamma$, IL-2 and TNF$\alpha$ and can drive B cell production of IgG2a to neutralize infective viral particles. This review will focus specifically on the role of CD4 cells in the immune response to influenza, an acute, localized respiratory viral infection. We suggest that CD4 cells act as direct effectors in protection against influenza, may contribute to immunopathology and generate functionally distinct memory subsets.
\end{abstract}

Keywords: anti-viral immunity, CD4 T cells, influenza, IFN- $\gamma$, memory T cells, cytotoxicity

Abbreviations: LN, lymph node; PR8, A/Puerto Rico/8/34; BAL, bronchoalveolar lavage; IFN, interferon; CFSE, carboxyfluorescein diacetate succinimydyl ester

\section{Introduction}

CD4 T lymphocytes can be subdivided into two groups based on the cytokine profiles they produce. Upon antigen encounter in the presence of IL-12 and IFN- $\gamma$, naive CD4 cells can differentiate into T helper 1 (Th1) cells that are characterized by the production of IFN- $\gamma$, IL-2 and TNF-a. Alternatively, antigen signaling in the presence of IL-4 induces the naive CD4 cell population to develop into Th2 effectors secreting IL-4, IL-5 and IL-13 [1]. Examples of Th2-associated responses include extracellular parasitic infections such as Schistosoma and Nippostrongylus and allergic and atopic diseases [2]. Viral infections are known to predominantly induce Th1 or Type 1 immunity that promotes the activation of CD8 T cells and macrophage functions and drives $B$ cell differentiation. Type 1 responses are also prevalent in some autoimmune disorders [2] and are a hallmark of effective anti-tumor responses [3]. Understanding the role of CD4 cells in various immune responses is paramount for designing effective therapies for malignancy and autoimmunity and for formulating better vaccines that promote cell-mediated immunity to infection. This review will focus on the importance of CD4 cells in a localized respiratory tract infection, influenza. Special emphasis is given to the comparison of the CD4 response to influenza in the lung with the response to systemic and persistent infections such as LCMV and murine gammaherpesvirus, respectively.
Several factors contribute to the immune response to viral infection including the nature of the pathogen itself, the route of infection, whether it is a chronic persisting, or acute infection and how the virus is presented to specific $\mathrm{T}$ cells for the adaptive response. Viruses are obligate intracellular parasites that replicate using host transcriptional and translational machinery. Influenza A viruses and the parainfluenza Sendai virus are negative strand RNA viruses that have limited infectivity due to the anatomical restriction of an enzyme necessary to cleave the viral surface proteins [4]. As such, replication is limited to the epithelial layer of the respiratory tract where, during the lytic phase of the virus life cycle, it causes tissue destruction. In contrast, lymphocytic choriomeningitis virus (LCMV) is considered a non-lytic RNA virus that can replicate in the host without causing major damage. Infection of mice with LCMV via tail vein injection induces a systemic infection with high viral titers in spleen and liver that is eventually resolved by the host immune response [5]. In situations where the host response is suppressed, LCMV infection can become more persistent with high viral titers but little tissue destruction [5]. Finally, some viruses such as herpeseviruses (EBV) and retroviruses (HIV) are cytopathic viruses that cause an initial lytic infection followed by a latent persistent infection [4]. 


\section{Complexity of the adaptive immune response to influenza}

Intranasal administration of influenza virus infects epithelial cells of the airways and is localized and confined to the respiratory tract. Infection with sublethal doses of virus results in an acute infection since normal mice mount a vigorous immune response that limits viral spread and promotes complete clearance from the lung within 7-10 days. Cytotoxic CD8 T cells play a major role in the clearance of influenza virus from the lungs via a class-I-dependent cytotoxic mechanism involving perforin and Fas [6]. Early work using class-Ideficient mice ( $\beta 2$ microglobulin $^{-/-}$), which lack CD8 cells, suggested that viral clearance of HKx31 influenza in the respiratory tract was delayed [7]. In mice infected with a highly pathogenic strain of influenza, A/Puerto Rico/8/34 (PR8), a lack of CD8 cells led to increased viral replication and eventual morbidity [8]. This latter result would seem to indicate that CD8 cells were absolutely required for viral clearance.

It has also been demonstrated that B cells and neutralizing antibodies can play an important role in immunity to influenza. B cell-deficient $\mu$ MT mice could clear HKx31 influenza with slightly delayed kinetics and normal CTL responses [9]. However, clearance of PR8 influenza was compromised in $\mu \mathrm{MT}$ mice and this led to increased morbidity compared to that of wildtype controls [10]. Thus, the relative requirements for CD8 cells versus B cells in the clearance of influenza may depend upon the pathogenicity and virulence of different strains [11, 12].

\section{Requirement for CD4 cells in anti-viral responses}

To investigate whether CD4 cells were absolutely required for clearance of virus, early work made use of depleting antibodies to CD4 [13] or gene targeted mice deficient in class II molecules [14, 15]. In anti-CD4 antibody treated mice, there was no difference in the CTL precursor frequency in the draining LN or the BAL in response to HKx31 infection [13]. Moreover, mice deficient in MHC class II molecules and thus devoid of most CD4 cells, demonstrated similar CTL activity compared to wild type mice and only a slight delay in viral clearance from the lungs [14]. In another report, the precursor CTL frequencies were four- to six-fold lower in class $\mathrm{II}^{-/-}$mice than in wild type counterparts; however, the lytic ability of CTL effectors at the peak of the anti-viral response was equivalent between CD4-deficient and wildtype mice [15]. It should also be noted that IL-2 and IFN- $\gamma$, as well as IL-4 and IL-5 production in the draining LN and BAL of CD4deficient mice was greatly reduced, suggesting that CD4 cells contribute to the immune response at the site of infection by the secretion of cytokines [15].

CD4-depleted mice could effectively clear PR8 virus, implying that CD4 cells were not required for elimination of virulent influenza strains [16]. However, the role of CD4 cells in response to influenza becomes more apparent when B cells and T helper cells are absent. $\mu \mathrm{MT}$ mice treated with anti-CD4 depleting antibodies cannot clear PR8 virus and show a high mortality to this virulent strain [16]. This suggests that CD8 cells alone cannot clear virus and indicates that CD4 cells may be important in helping to sustain the cytotoxic $\mathrm{T}$ cell response. In a similar set of experiments, using B cell-deficient mice treated with anti-CD8 depleting antibodies, the remaining $\mathrm{CD} 4$ response alone was ineffective at clearing virus and mice succumbed to an otherwise sublethal dose of HKx31 [17] or PR8 [10]. These data indicated that CD4 cells alone could not effectively clear virus and suggested that their main purpose in anti-viral immunity may be to provide help for antibody production $[10,17]$. Therefore, the results from cell depletion studies indicated that CD4 cells may only participate indirectly to provide help for CD8 and B cells.

Table 1, adapted from Gerhard [12], indicates that for a virulent strain of influenza, neither CD8 cells, CD4 cells, or B cells alone can effectively clear virus and mice succumb to what would otherwise be a sublethal dose of virus. However, if combinations of cells are present, mice can clear infection with slightly delayed kinetics and increased survival. Therefore, the immune response to pathogenic strains of influenza requires a complex interplay between cytotoxic $\mathrm{T}$ cells, antibody secreting $\mathrm{B}$ cells and cytokine secreting CD4 cells.

A role for CD4 $\mathrm{T}$ cells is also observed in CD8 responses to LCMV. During acute LCMV infection, CD8 cytotoxic cells are required as mice deficient in CD8 cells cannot effectively clear virus [18]. However, the CD8 response appears in part to rely on CD4 cells since CD4deficient mice demonstrated delayed viral clearance and a decline in CTL activity late in the primary response and into the secondary response [18]. Furthermore, CTL activity and CD8 memory responses were impaired in class-II-deficient mice leading to a recrudescence of viremia 2 months after initial infection [19]. Thus, in the primary response to both localized and systemic infections,

Table 1. Adapted from Gerhard, demonstrating the complexity of the immune response to virulent strains of influenza.

\begin{tabular}{ccrrr}
\hline CD8 & CD4 & B cells & Clearance (days) & Survival (\%) \\
\hline+ & + & + & $7-10$ & 100 \\
- & + & + & $10-14$ & 100 \\
- & + & - & $>20$ & 0 \\
- & - & + & $>20$ & 0 \\
+ & - & - & $>14$ & 20 \\
+ & + & - & $10-14$ & $35-85$ \\
+ & - & + & $10-14$ & 90 \\
- & - & - & $>20$ & 0
\end{tabular}

Each cell type of the adaptive immune response was depleted via treatment with antibody, using genetically altered mice, or a combination of both. As shown in rows 3, 4 and 5 neither CD8, CD4 nor B cells alone can effectively clear virus or promote survival of mice given A/Puerto Rico/8/34. 
CD4 cells appear to be dispensible as long as CD8 cells and B cells are present. In contrast, CD4 cells are required in the immune response to such chronic infections as gamma herpesvirus [20] and chronic infection with LCMV [21]. Moreover, CD4 cells are important for the maintenance of CD8 cytolytic responses and the transition to the memory phase in both acute, localized infections such as influenza [22] and systemic LCMV infections $[19,23]$.

\section{Initiation of the response: DCs activate CD4 helper cells}

Activation of naive CD4 cells requires antigen specific signaling through the $\mathrm{T}$ cell receptor (TCR) as well as signaling through co-stimulatory molecules. It is likely that lymph nodes and perhaps spleen provide the spatial organization and appropriate chemokine environment to bring together professional APCs and naive CD4 cells during priming of an anti-viral immune response [24, 25]. Dendritic cells (DC) at the site of infection can engulf viral particles, mature in response to inflammatory signals, migrate to draining LN and present peptide antigen in the context of class II molecules to activate naive virusspecific CD4 cells [26]. It has been well-documented that the skin contains a web of immature DC termed Langerhans cells that can migrate rapidly to draining LN in response to a variety of inflammatory stimuli [27]. It has recently been appreciated that the respiratory tract contains a similar network of resident DC that migrate to respiratory LN under steady state conditions [28, 29]. Using intranasal administration of CFSE followed by influenza infection, it was shown that a high percentage of $\mathrm{CD} 11 \mathrm{c}^{+}$/ $\mathrm{CFSE}^{+}$cells accumulated in the draining LN that peaked $18 \mathrm{~h}$ after infection [29]. Interestingly, DC accumulation declined quickly and the ability of these cells to migrate to an additional inflammatory stimulus was diminished [29]. This suggests that DC migration in response to influenza infection is transient and it remains to be determined if other cell types transport antigen to the draining $\mathrm{LN}$ after the initial wave of DC migration. In addition, it is not known what effect chronic infections may have on DC migration or if an immune response to a secondary infection is compromised due to the short-lived nature of the $\mathrm{DC}$ response.

\section{Tracking anti-viral CD4 responses directly: techniques}

Although CD4 cells themselves may not have a primary role in the clearance of acute viral infections, their role in maintaining CD8 T cell memory and B cell responses is well-established in many models [12, 30, 31]. Most of the early work quantitating the frequencies of anti-viral CD4 responses made use of limiting dilution analysis (LDA) and IFN- $\gamma$ ELISPOT assays [4, 32]. Frequencies of influenza reactive CD4 cells were estimated by LDA analysis to be in the order of approximately 1 in 300 in the spleen at the peak of the immune response [17]. This study did not analyze the response in the lung or the BAL, where the infection is localized and high numbers of CD4 cells are recruited $[33,34]$. In a similar respiratory infection, the frequencies of $\mathrm{CD} 4$ cells reactive to Sendai virus were enumerated and found to be equivalent to those obtained in the influenza system [35]. Comparably, in response to a systemic infection with LCMV, the frequency of IFN- $\gamma$ secreting CD4 cells was shown to be about 1 in 200 against virally infected targets and 1 in 40 for the dominant class II peptide [36, 37]. In all viral infections that are resolved after an acute phase, the frequency of virus-specific CD4 cells drops to approximately 1 in 500 to 1 in 1000 in the memory phase [9, 35-37]. Conversely, during persistent infection with a murine gammaherpesvirus, CD4 cell frequencies were shown to remain high even after many months [38].

With the advent of new technologies, it is now possible to track virus-specific CD8 and CD4 cells directly using complexes of MHC class I or class II molecules loaded with specific peptides $[39,40]$. With this technique, it is possible to quantitate $\mathrm{CD} 4$ responses directly without culturing cells and estimating precursor frequencies. It has been demonstrated that the dominant CD4 response to LCMV is directed toward a glycoprotein peptide 6180 , but the frequency of cells reactive to this epitope has been underestimated using LDA and ELISPOT assays. While ELISPOT assays have estimated the frequency of CD4 cells reactive to $\mathrm{GP}_{61-80}$ to be approximately 1 in 40 , multimer analysis has enumerated the dominant CD4 response to be 1 in 9 [41]. Thus, multimers can detect cells that have expanded in response to infection; however, there may be a disparity between the number of antigen reactive cells and the number of terminally differentiated effector cells capable of secreting cytokines. Importantly, this technology has provided insight to the differences in expansion, contraction and memory generation between virus-specific CD8 and CD4 responses. For both LCMV [41] and Sendai infection [42], it has been observed that the CD8 expansion or burst size is greater than the CD4 expansion phase. Virus-specific CD4 cells contract with different kinetics than CD8 cells and the frequency of CD4 cells detected in the memory pool is much lower (1 in 500) than the frequency of CD8 cells (1 in 12) [41]. In our own experiments using adoptive transfer of TCR Tg CD8 and CD4 cells reactive to hemagglutinin peptides, we have found that CD8 numbers in the lung and BAL exceeded the numbers of CD4 cells at the site of infection, regardless of the input cell numbers (Powell, et al. manuscript submitted). The mechanisms regulating the numbers of CD8 and CD4 cells have yet to be elucidated, however, it has been suggested that differences in survival factors such as Bcl-2 could be responsible [41]. Alternatively, cytokines that regulate homeostatic proliferation and survival such as IL-2, IL-7 and IL-15 may affect CD8 cells differently than CD4 cells [32, 43, 44]. 
There exists to date various multimeric reagents to detect CD4 responses to LCMV [41], ovalbumin, pigeon cytochrome C [40], and mycobacterial antigens [45]; however, there seems to be a paucity of CD4 epitopes defined for influenza. In BALB/c mice infected with PR8 virus, Gerhard and co-workers [46] have identified CD4 $\mathrm{T}$ cell clones that are reactive to purified matrix, nucleoprotein, hemagglutinin and neuraminidase proteins. Using synthetic peptides, eight distinct epitopes were identified in the hemagglutinin protein of PR8 that were recognized by $\mathrm{T}$ cell clones [47]. T cell receptors from two of these clones have been used to generate TCR Tg mice that recognize either $\mathrm{HA}_{110-120}$ [48] or $\mathrm{HA}_{126-138}$ [49]. Despite the availability of some reagents, the low frequency of CD4 cells and low affinity of class II multimers for peptide makes detection of the primary response difficult without first priming in the presence of peptide and adjuvant [42].

To circumvent some of these difficulties, our laboratory employs an adoptive transfer system in which CD4 cells from TCR transgenic mice that recognize a peptide 126138 in the hemagglutinin (HA) protein of PR8 influenza virus [49] are transferred into Thy 1 disparate BALB/ c mice. After sublethal infection, CFSE labeled TCR Tg CD4 cells can be visualized for division and effector generation using four color flow cytometry with antibodies to CD4, Thy1 and various surface markers [34]. This allows the characterization of the early events in CD4 cell proliferation and activation in response to infection that cannot be appreciated using tetramer or IFN- $\gamma$ ELISPOTs due to the low frequency of the initial CD4 population.

\section{Primary CD4 responses to influenza}

HA-specific CD4 TCR Tg cells were adoptively transferred into normal BALB/c mice and subsequently challenged with a sublethal dose of PR8. Our data indicate that there was a vigorous CD4 T cell response to influenza initiating in the draining LN followed by the spleen and peaking in the lung and bronchoalveolar lavage (BAL) at 6-7 days post-infection [34]. This response was dominated by a Th1 cytokine profile as IL-2 was made by Tg CD4 cells in the draining LN, but only IFN-ץ and TNF- $\alpha$ was produced by terminally differentiated CD4 effector cells in the lung and BAL. CD4 T cell proliferation was initiated in the draining mediastinal LN where cells in each round of cell division could be observed. Only the cells that had divided completely and had differentiated to an effector cell (as defined by phenotype and cytokine secretion) could be found in the lung and BAL. It is not yet clear how the vigorous primary CD4 response and the production of IFN- $\gamma$ by recruited CD4 effectors contributes to protection in the lung. Furthermore, it is not known how CD4 effectors contribute to the generation of protective memory at the site of infection and these questions are the focus of current efforts.

\section{CD4 effector mechanisms and protection}

The predominance of a Th1 response characterized by secretion of IFN- $\gamma$ led to the investigation of this cytokine in the immune response to influenza. Infection of mice with a targeted disruption of the IFN- $\gamma$ gene demonstrated increases in type 2 cytokine production and increases in influenza-specific IgG1 antibody secretion [50]. However, cytotoxic T cell responses in these mice were comparable to wildtype controls. Interestingly, CD4 cell clones isolated from IFN- $\gamma^{-/-}$mice and restimulated in vitro could protect against lethal challenge with influenza via a cytolytic mechanism [50]. It has also been demonstrated that virally infected classI-deficient mice have CD4 cells capable of lysing Sendai or LCMV-infected targets [11], suggesting that CD4 cells can compensate for the lack of IFN-y or CD8 cells by becoming killers themselves. The importance of cytolytic CD4 cells in the response to influenza remains controversial since experiments using bone marrow chimeras with class $\mathrm{II}^{-/-}$epithelial cells demonstrated that CD4 cells did not have to directly recognize infected epithelium in order to exert effector function [11]. In contrast,

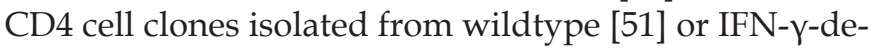
ficient mice [50] could protect against a lethal inoculation with influenza that depended not on their cytokine profile, but their ability to lyse virally infected targets.

We found that TCR Tg CD4 effectors specific for HA peptide that are recovered from the lung and BAL of infected mice produce high amounts of IFN- $\gamma$ ex vivo, but undetectable levels of Th2 cytokines such as IL-4 ([34] and Brown, unpublished observations). To further dissect the mechanism of CD4 mediated protection to lethal influenza infection we have utilized a monoclonal CD4 cell population from TCR Tg mice deficient in IFN- $\gamma$ as well as polyclonal CD4 effectors from sublethally infected BALB / c IFN- $\gamma^{-/-}$mice. Table 2 shows that protection mediated by in vivo generated, polyclonal CD4 effector cells was dependent on IFN- $\gamma$, however, protection mediated by unfractionated lung cells did not require IFN- $\gamma$. In a separate set of experiments, influenza-specific wildtype and IFN- $\gamma$-deficient TCR Tg CD4 cells were stimulated in vitro with HA peptide and Th1 polarizing cytokines. These monoclonal populations were then transferred i.v. to BALB/c mice that were then infected with a lethal dose of PR8 and monitored for survival (Table 2). Surprisingly, IFN- $\gamma$ was not required for CD4-mediated protection by a monoclonal, in vitro stimulated population of effectors. Furthermore, both wild type and IFN- $\gamma$ deficient in vitro generated effectors were cytolytic suggesting an alternate mechanism of protection in vivo ( Table 2). We suggest that one possiblility to explain these findings is that in vivo generated effectors may not be cytolytic and are more dependent on IFN- $\gamma$ for effector function, whereas in vitro generated effectors have compensated for the lack of IFN by acquiring high CTL activity. In a similar study, 
Table 2. Protection mediated by in vivo generated CD4 effectors is dependent on IFN- $\gamma$

\begin{tabular}{|c|c|c|c|c|}
\hline Isolation & Effectors & $\begin{array}{c}\mathrm{IFN}-\gamma \\
\text { production }\end{array}$ & $\begin{array}{c}\text { Survival } \\
(\%)\end{array}$ & $\begin{array}{c}\text { Cytolytic } \\
\text { activity }\end{array}$ \\
\hline \multirow[t]{3}{*}{ In vivo, total lung } & Polyclonal WT & Yes & 100 & $\mathrm{ND}^{*}$ \\
\hline & Polyclonal IFN- $\gamma-/-$ & No & 100 & ND \\
\hline & None & - & 20 & - \\
\hline \multirow[t]{3}{*}{ In vivo, CD4 cells } & Polyclonal WT & Yes & 100 & ND \\
\hline & Polyclonal IFN- $\gamma-/-$ & No & 20 & ND \\
\hline & None & - & 0 & - \\
\hline \multirow[t]{3}{*}{ In vitro, Th1 } & TCR Tg WT & Yes & 100 & Yes $(++)$ \\
\hline & TCR Tg IFN- $\gamma-/-$ & No & 100 & Yes $(+++)$ \\
\hline & None & - & 0 & - \\
\hline
\end{tabular}

Total lung cells or CD4 cells from lung and draining LN were isolated from wildtype BALB/c or IFN- $\gamma$-deficient BALB/c mice 7 days after a sublethal infection with influenza PR8 (polyclonal WT or IFN- $\gamma^{-/-}$). TCR Tg CD4 cells from wildtype or IFN- $\gamma^{-}$-deficient mice were stimulated in vitro with B cell blasts and specific peptide in the presence of IL-2, IL-12 and anti-IL-4 (in vitro Th1). Cells were transferred to normal BALB/c mice that were subsequently infected with a 5LD ${ }_{50}$ dose of PR8 and monitored for survival. BALB/c mice that did not receive cells served as a control. Wildtype or IFN- $\gamma$-deficient Th1 effectors were tested for cytolytic activity in an in vitro JAM assay using peptide pulsed A20 cells or unpulsed A20 as a negative control.

it was demonstrated that Sendai-specific memory CD4 cells isolated from BAL fluid and transferred to naive mice could reduce viral load after Sendai infection [52]. Therefore, virus-specific CD4 cells can confer protection in both intact (Brown, manuscript in preparation) [51] and $\beta 2 \mathrm{~m}$-deficient mice [52]. This strongly suggests that CD4 cells may exert direct protective effects in response to influenza. What is not known is whether these effectors are directly cytolytic in vivo, or whether they recruit other host cells to the site of infection due to rapid expression of cytokines and chemokines, or confer protection by a combination of mechanisms.

The balance between viral clearance and tissue destruction may, in some cases lead to immunopathology. This has been demonstrated in the immune response to influenza, where CD8 effectors unable to secrete IFN$\gamma$ caused more lung damage than Tc1 effectors capable of producing IFN- $\gamma$ [53]. Similarly, CD8 cells in chronic LCMV infection were shown to induce wasting disease in IFN- $\gamma$-deficient, CD4-depleted mice [21]. This suggests that IFN- $\gamma$ secretion by CD8 cells exerts a protective effect during immune-mediated damage in the lung and other organs. Indeed, damage mediated by Tc1 IFN$\mathrm{Y}^{-/-}$and Tc2 IFN- $\mathrm{\gamma}^{-/-}$effectors included increases in eosinophilia and necrotizing lesions [53]. It is not known to what extent CD4 cells contribute to lung immunopathology after influenza infection, however, it is known that Th2 cells do not protect against lethal influenza infection [51] and the reason for this may be due to increased pathology mediated by the type 2 cytokines, IL-4 and IL-5 leading to eosinophilia. Recently, it has been shown that blocking the OX40-OX40L costimulatory pathway can lead to decreased $\mathrm{T}$ cell influx into the lungs and diminished immunopathology after influenza infection [54]. It remains to be determined if CD4 and CD8 cells contribute equally to immunopathology after infection with more virulent strains of influenza. Further, it is not known where influenza-specific CD4 cells localize in the lung parenchyma and what other types of cells they contact within the lung. If the primary role of CD4 cells is to help promote the antibody response, then why do such large numbers of activated CD4 effectors traffic to the lung and BAL? Is this a consequence of inflammation and recruitment of cells due to their activation status alone, or is there evidence for CD4/CD8 and or CD4/B cell interactions within lung tissue?

\section{Memory CD4 responses to influenza}

It has been demonstrated, using multimers, that the contraction phase in the CD4 compartment is more pronounced than in the CD8 population, leading to a decline in CD4 cell numbers in the memory phase in LCMV [41] and Sendai infection [42]. Tracking CD4 cells into the memory phase after influenza infection has been technically difficult due to the low numbers of CD4 cells that persist into memory, and the availability of reagents to detect low frequencies of cells. In our studies using the adoptive transfer system, we have shown a similar pattern to what has been observed in LCMV and Sendai infection. Specifically, influenza reactive CD4 cells demonstrate an increased contraction phase compared to influenza-specific CD8 cells at the site of infection, namely the lung parenchyma and the airways (Powell, et al., manuscript submitted; Roman, unpublished observations). In addition, the frequency of CD4 cells that persist into memory is lower than the frequency of CD8 cells in non-lymphoid sites (lung and BAL). Interestingly, the frequency of CD4 cells in the memory phase in the lymphoid sites (spleen and LN) more closely resemble the frequency of CD8 memory cells.

The primary CD4 response to influenza is comprised of a heterogeneous mix of effector populations in which the effector cells in the lung and BAL show a more activated phenotype while CD4 effector cells remaining in the lymphoid organs are less differentiated and mainly 
produce IL-2 [34]. After viral clearance, effector cells representing all stages of differentiation can give rise to corresponding heterogeneous memory populations. CD4 memory cells detected in the LN and spleen demonstrate high levels of CD62L and CCR7 while CD4 memory cells detected in the lung more than 30 days post-infection are small resting cells that express low levels of CD62L and CCR7 and high CD43 (Roman, et al., in preparation). The generation of heterogeneous memory subsets in different organs suggests that functional CD4 memory could be different based on distinct anatomical localization. The CD4 memory response to Sendai virus is also heterogeneous [42]. Phenotypic markers such as CD43 and CD11a are differentially expressed on tetramer positive cells in the lung parenchyma compared to the airways and tet ${ }^{+}$ cells in the lung demonstrate a highly activated phenotype compared to cells in the lymphoid organs [42]. Taken together, these data suggest that there are multiple, phenotypically diverse subsets of memory CD4 cells that are likely to function differently. In particular, CD4 memory subsets in the lung are poised for immediate response to reinfection in the respiratory tract while CD4 cells in the lymphoid organs may be recruited later in the response. An interesting aspect of these studies is that the decay of CD4 memory cells in the lungs is faster than in the periphery suggesting a temporal, as well as phenotypic difference in long lasting CD4 immunity to infection. This may have important implications for vaccine development and the functions of CD4 memory subsets are currently being investigated in our laboratory.

\section{Summary and concluding remarks}

CD4 cells are an important component of the anti-viral response to local and systemic infections. Although early studies using CD4-depleted mice have suggested that CD4 cells were not absolutely required in the primary response, more recent studies have determined that CD4 cells were necessary for long lasting, effective CD8 memory. In addition, the primary CD4 $\mathrm{T}$ cell response to influenza infection, albeit smaller in magnitude than the CD8 response, was shown to involve robust expansion, Th1 differentiation and migration to the site of infection. CD4 effector cells can also promote survival to a lethal dose of influenza infection and may contribute to immune-mediated pathology. Finally, the heterogeneity in the CD4 population observed in the primary response is recapitulated in the memory phase after the infection has been cleared. Thus, we consider the possibility that CD4 effector $\mathrm{T}$ cells and memory contribute to immunity to influenza via multiple mechanisms that are just beginning to be appreciated. These may include their classic contribution as helpers during the generation of flu-specific CD8 CTL and antibody producing B cells; and also the possibility that CD4 effectors in the lung may directly mediate viral clearance by IFN- $\gamma$-dependent mechanisms and/or by direct cytolytic effects on infected cells.

\section{Acknowledgements}

This work was supported by public health services grants T32-AI49823 (DMB) and PO1-HL63925. The authors would like to thank Drs. Laura Haynes and Roslyn Kemp for critical review of this manuscript and Kim Sorrell for technical assistance.

\section{References}

1. A. K. Abbas, K. M. Murphy and A. Sher, Functional diversity of helper T lymphocytes. Nature 383 (1996), pp. 787-793.

2. T. R. Mosmann and S. Sad, The expanding universe of T-cell subsets: Th1, Th2 and more. Immunol. Today 17 (1996), pp. 138-146.

3. D. M. Pardoll, Inducing autoimmune disease to treat cancer. Proc. Natl Acad. Sci. U. S. A. 96 (1999), pp. 5340-5342.

4. P. C. Doherty and J. P. Christensen, Accessing complexity: the dynamics of virus-specific T cell responses. Annu. Rev. Immunol. 18 (2000), pp. 561-592.

5. Ahmed R. Immunity to viruses. In: Paul WB, Paul WBs, editors. Fundamental immunology. Philadelphia, PA: Lippincott-Raven; 1999.

6. D. J. Topham, R. A. Tripp and P. C. Doherty, CD8+ T cells clear influenza virus by perforin or Fas-dependent processes. J. Immunol. 159 (1997), pp. 5197-5200.

7. M. Eichelberger, W. Allan, M. Zijlstra, R. Jaenisch and P. C. Doherty, Clearance of influenza virus respiratory infection in mice lacking class I major histocompatibility complex-restricted CD8+ T cells. J. Exp. Med. 174 (1991), pp. 875-880.

8. B. S. Bender, T. Croghan, L. Zhang and P. A. Small, Jr. , Transgenic mice lacking class I major histocompatibility complexrestricted $\mathrm{T}$ cells have delayed viral clearance and increased mortality after influenza virus challenge. J. Exp. Med. 175 (1992), pp. 1143-1145.

9. D. J. Topham, R. A. Tripp, A. M. Hamilton-Easton, S. R. Sarawar and P. C. Doherty, Quantitative analysis of the influenza virus-specific CD4+ T cell memory in the absence of B cells and Ig. J. Immunol. 157 (1996), pp. 2947-2952.

10. K. Mozdzanowska, M. Furchner, K. Maiese and W. Gerhard, CD4+ T cells are ineffective in clearing a pulmonary infection with influenza type A virus in the absence of B cells. Virology 239 (1997), pp. 217-225.

11. P. C. Doherty, D. J. Topham, R. A. Tripp, R. D. Cardin, J. W. Brooks and P. G. Stevenson, Effector CD4+ and CD8+ T-cell mechanisms in the control of respiratory virus infections. Immunol. Rev. 159 (1997), pp. 105-117.

12. W. Gerhard, The role of the antibody response in influenza virus infection. Curr. Top. Microbiol. Immunol. 260 (2001), pp. 171-190.

13. W. Allan, Z. Tabi, A. Cleary and P. C. Doherty, Cellular events in the lymph node and lung of mice with influenza. Consequences of depleting CD4+ T cells. J. Immunol. 144 (1990), pp. 3980-3986.

14. H. Bodmer, G. Obert, S. Chan, C. Benoist and D. Mathis, Environmental modulation of the autonomy of cytotoxic T lymphocytes. Eur. J. Immunol. 23 (1993), pp. 1649-1654.

15. R. A. Tripp, S. R. Sarawar and P. C. Doherty, Characteristics of the influenza virus-specific CD8+ T cell response in mice homozygous for disruption of the H-2lAb gene. J. Immunol. 155 (1995), pp. 2955-2959.

16. K. Mozdzanowska, K. Maiese and W. Gerhard, Th cell-deficient mice control influenza virus infection more effectively than Th- and B cell-deficient mice: evidence for a Th-independent contribution by B cells to virus clearance. J. Immunol. 164 (2000), pp. 2635-2643.

17. D. J. Topham and P. C. Doherty, Clearance of an influenza A 
virus by CD4+ T cells is inefficient in the absence of B cells. J. Virol. 72 (1998), pp. 882-885.

18. J. P. Christensen, O. Marker and A. R. Thomsen, The Role of CD4+ T cells in cell mediated immunity to LCMV: studies in MHC class I and class II deficient mice. Scand. J. Immunol. 40 (1994), pp. 373-382.

19. A. R. Thomsen, J. Johansen, O. Marker and J. P. Christensen, Exhaustion of CTL memory and recrudescence of viremia in lymphocytic choriomeningitis virus infected MHC class II deficient and B cell deficient mice. J. Immunol. 157 (1996), pp. 3074-3080.

20. J. P. Christensen, R. D. Cardin, K. C. Branum and P. C. Doherty, CD4(+) T cell-mediated control of a gamma-herpesvirus in B cell-deficient mice is mediated by IFN-gamma. Proc. Natl. Acad. Sci. U. S. A. 96 (1999), pp. 5135-5140.

21. J. P. Christensen, C. Bartholdy, D. Wodarz and A. R. Thomsen, Depletion of CD4+ T cells precipitates immunopathology in immunodeficient mice infected with a noncytocidal virus. J. Immunol. 166 (2001), pp. 3384-3391.

22. G. T. Belz, D. Wodarz, G. Diaz, M. A. Nowak and P. C. Doherty, Compromised influenza virus-specific CD8(+)-T-cell memory in CD4(+)-T-cell-deficient mice. J. Virol. 76 (2002), pp. 12388-12393.

23. M. G. von Herrath, M. Yokoyama, J. Dockter, M. B. Oldstone and J. L. Whitton, CD4-deficient mice have reduced levels of memory cytotoxic $\mathrm{T}$ lymphocytes after immunization and show diminished resistance to subsequent viral challenge. $J$. Virol. 70 (1996), pp. 1072-1079.

24. E. J. Kunkel and E. C. Butcher, Chemokines and the tissue-specific migration of lymphocytes. Immunity 16 (2002), pp. 1-4.

25. A. A. Itano and M. K. Jenkins, Antigen presentation to naive CD4 T cells in the lymph node. Nat. Immunol. 4 (2003), pp. 733-739.

26. F. R. Carbone and W. R. Heath, The role for dendritic cell subsets in immunity to viruses. Curr. Opin. Immunol. 15 (2003), pp. 416-420.

27. J. Banchereau, F. Briere, C. Caux, J. Davoust, S. Lebecque, Y. J. Liu et al. , Immunobiology of dendritic cells. Annu. Rev. Immunol. 18 (2000), pp. 767-811.

28. P. A. Stumbles, J. W. Upham and P. G. Holt, Airway dendritic cells: co-ordinators of immunological homeostasis and immunity in the respiratory tract. Apmis 111 (2003), pp. 741-755.

29. K. L. Legge and T. J. Braciale, Accelerated migration of respiratory dendritic cells to the regional lymph nodes is limited to the early phase of pulmonary infection. Immunity 18 (2003), pp. 265-277.

30. S. A. Kalams and B. D. Walker, The critical need for CD4 help in maintaining effective cytotoxic T lymphocytes responses. J. Exp. Med. 188 (1998), pp. 2199-2204.

31. D. L. Woodland, Cell-mediated immunity to respiratory virus infections. Curr. Opin. Immunol. 15 (2003), pp. 430-435.

32. J. K. Whitmire and R. Ahmed, The economy of T-cell memory: CD4+ recession in times of CD8+ stability?. Nat. Med. 7 (2001), pp. 892-893.

33. N. Baumgarth and A. Kelso, Functionally distinct T cells in three compartments of the respiratory tract after influenza virus infection. Eur. J. Immunol. 26 (1996), pp. 2189-2197.

34. E. Roman, E. Miller, A. Harmsen, J. Wiley, U. H. Von Andrian, G. Huston et al. , CD4 effector T cell subsets in the response to influenza: heterogeneity, migration, and function. J. Exp. Med. 196 (2002), pp. 957-968.

35. D. J. Topham and P. C. Doherty, Longitudinal analysis of the acute Sendai virus-specific CD4+ T cell response and memory. J. Immunol. 161 (1998), pp. 4530-4535.

36. J. K. Whitmire, M. S. Asano, K. Murali-Krishna, M. Suresh and R. Ahmed, Long term CD4 Th1 and Th2 memory following acute lymphocytic choriomeningitis virus infection. J. Virol. 72 (1998), pp. 8281-8288.
37. C. Kamperschroer and D. G. Quinn, Quantification of epitope specific MHC class II restricted T cells following lymphocytic choriomeningitis virus infection. Cell. Immunol. 193 (1999), pp. 134-146.

38. J. P. Christensen and P. C. Doherty, Quantitative analysis of the acute and long-term $\mathrm{CD} 4(+) \mathrm{T}$-cell response to a persistent gammaherpesvirus. J. Virol. 73 (1999), pp. 4279-4283.

39. J. D. Altman, P. A. Moss, P. J. Goulder, D. H. Barouch, M. G. McHeyzer-Williams, J. I. Bell et al. , Phenotypic analysis of antigen-specific T lymphocytes. Science 274 (1996), pp. 94-96.

40. P. Y. Arnold, K. M. Vignali, T. B. Miller, N. L. La Gruta, L. S. Cauley, L. Haynes et al. , Reliable generation and use of MHC class II:gamma2aFc multimers for the identification of antigen-specific CD4(+) T cells. J. Immunol. Methods 271 (2002), pp. 137-151.

41. D. Homann, L. Teyton and M. B. Oldstone, Differential regulation of antiviral T-cell immunity results in stable CD8+ but declining CD4+ T-cell memory. Nat. Med. 7 (2001), pp. 913-919.

42. L. S. Cauley, T. Cookenham, T. B. Miller, P. S. Adams, K. M. Vignali, D. A. Vignali et al. , Cutting edge: virus-specific CD4+ memory $\mathrm{T}$ cells in nonlymphoid tissues express a highly activated phenotype. J. Immunol. 169 (2002), pp. 6655-6658.

43. D. F. Tough and J. Sprent, Turnover of naive- and memoryphenotype T cells. J. Exp. Med. 179 (1994), pp. 1127-1135.

44. R. A. Seder and R. Ahmed, Similarities and differences in CD4+ and CD8+ effector and memory T cell generation. Nat. Immunol. 4 (2003), pp. 835-842.

45. G. M. Winslow, A. D. Roberts, M. A. Blackman and D. L. Woodland, Persistence and turnover of antigen-specific CD4 T cells during chronic tuberculosis infection in the mouse. J. Immunol. 170 (2003), pp. 2046-2052.

46. J. L. Hurwitz, C. J. Hackett, E. C. McAndrew and W. Gerhard, Murine Th response to influenza virus: recognition of hemagglutinin, neuraminidase, matrix and nucleoproteins. J. Immunol. 134 (1985), pp. 1994-1998.

47. W. Gerhard, A. M. Haberman, P. A. Scherle, A. H. Taylor, G. Palladino and A. J. Caton, Identification of eight determinants in the hemagglutinin molecule of influenza virus A/PR/8/34 (H1N1) which are recognized by class II restricted T cells from BALB/c mice. J. Virol. 65 (1991), pp. 364-372.

48. J. Kirberg, A. Baron, S. Jakob, A. Rolink, K. Karjalainen and H. von Boehmer, Thymic selection of CD8+ single positive cells with a class II major histocompatibility complex-restricted receptor. J. Exp. Med. 180 (1994), pp. 25-34.

49. B. Scott, R. Liblau, S. Degermann, L. A. Marconi, L. Ogata, A. J. Caton et al. , A role for non-MHC genetic polymorphism in susceptibility to spontaneous autoimmunity. Immunity $\mathbf{1}$ (1994), pp. 73-82.

50. M. B. Graham, D. K. Dalton, D. Giltinan, V. L. Braciale, T. A. Stewart and T. J. Braciale, Response to influenza infection in mice with a targeted disruption in the interferon gamma gene. J. Exp. Med. 178 (1993), pp. 1725-1732.

51. M. B. Graham, V. L. Braciale and T. J. Braciale, Influenza virus-specific CD4+ T helper type $2 \mathrm{~T}$ lymphocytes do not promote recovery from experimental virus infection. J. Exp. Med. 180 (1994), pp. 1273-1282.

52. R. J. Hogan, W. Zhong, E. J. Usherwood, T. Cookenham, A. D. Roberts and D. L. Woodland, Protection from respiratory virus infections can be mediated by antigen- specific CD4(+) T cells that persist in the lungs. J. Exp. Med. 193 (2001), pp. 981-986.

53. J. A. Wiley, A. Cerwenka, J. R. Harkema, R. W. Dutton and A. G. Harmsen, Production of interferon-gamma by influenza hemagglutinin-specific CD8 effector T cells influences the development of pulmonary immunopathology. Am. J. Pathol. 158 (2001), pp. 119-130.

54. I. R. Humphreys, G. Walzl, L. Edwards, A. Rae, S. Hill and T. Hussell, A critical role for OX40 in T cell-mediated immunopathology during lung viral infection. J. Exp. Med. 198 (2003), pp. 1237-1242. 